\title{
Comportamento da variabilidade da frequência cardíaca e da capacidade funcional de acordo com o tempo de transplante renal
}

\author{
Behavior of variability of heart rate and functional capacity \\ according to renal transplant time
}

\author{
Camila Carolina Alves Andrade"; Tuíra Oliveira Maia²; Shirley Dias Bezerra²; Lívia Gomes \\ da Rocha²; Helga Cecília Muniz de Souza ${ }^{3}$; Patrícia Érika de Melo Marinho4 \\ 1 Programa de Residência Multiprofissional Integrada em Saúde do Hospital das Clínicas, Universidade Federal de Pernambuco - \\ UFPE. Recife, PE - Brasil. \\ 2 Laboratório de Fisioterapia Cardiopulmonar, Departamento de Fisioterapia, Universidade Federal de Pernambuco - UFPE. Recife, \\ PE - Brasil. \\ 3 Hospital das Clínicas, Universidade Federal de Pernambuco - UFPE. Recife, PE - Brasil. \\ 4 Departamento de Fisioterapia, Universidade Federal de Pernambuco - UFPE. Recife, PE - Brasil. \\ Endereço para correspondência: \\ Patrícia Érika M Marinho \\ Departamento de Fisioterapia, Universidade Federal de Pernambuco - UFPE \\ Avenida Jornalista Aníbal Fernandes, s/n - Cidade Universitária. \\ 50740-560 - Recife - PE [Brasil] \\ patmarinho@yahoo.com.br \\ patricia.marinho@ufpe.br
}

\begin{abstract}
Resumo
Introdução: Desequilíbrio autonômico, com aumento da atividade simpática e redução da parassimpática, pode ocorrer no transplantado renal, representando forte indicador de risco cardíaco. Objetivo: Avaliar a variabilidade da frequência cardíaca (VFC) e a capacidade funcional dos transplantados renais de acordo com o tempo de transplante renal. Métodos: Série de casos envolvendo transplantados renais divididos em grupos de acordo com a mediana do tempo de transplante renal (158 meses). Foram avaliados a VFC através do Holter por 24 horas, o nível de atividade física (IPAQ) e o desempenho funcional (teste de caminhada de 6 minutos). Resultados: Os indivíduos comportaram-se diferentemente em relação à VFC e à capacidade funcional. No entanto, aqueles com maior tempo de transplante apresentaram maior VFC, eram menos ativos e variaram mais no desempenho funcional. Conclusão: $O$ presente estudo constata a presença de diferenças individuais na VFC e no desempenho funcional entre os transplantados renais de acordo com o tempo de realização do TX.
\end{abstract}

Descritores: Insuficiência Renal Crônica; Transplante de Rim; Sistema Nervoso Autônomo; Frequência Cardíaca; Uremia.

\begin{abstract}
Introduction: Autonomic imbalance, with increased sympathetic activity and reduction of parasympathetic activity, may occur in the renal transplantation patient, representing a strong indicator of cardiac risk. Objective: To assess heart rate variability (HRV) and functional capacity of kidney transplantation recipients according to the time of transplantation. Methods: A case series involving renal transplant recipients divided into groups according to the median of kidney transplantation time (158 months). HRV was evaluated through 24-hour Holter monitoring, physical activity level (IPAQ) and functional performance (6-minute walk test). Results: The individuals behaved differently in relation to HRV and functional capacity. Those with longer transplantation had higher HRV, were less active and presented more diverse functional performance. Conclusion: The present study notes the presence of individual differences in HRV and functional performance between renal transplants according to the time of TX
\end{abstract}

Keywords: Renal Insufficiency, Chronic; Kidney Transplantation; Autonomic Nervous System; Heart Rate; Uremia. 


\section{Introdução}

A disfunção na modulação autonômica do sistema nervoso autônomo (SNA) representa importante indicador de saúde, da análise de funcionamento fisiológico e de fator prognóstico para os pacientes com doença renal crônica e transplantados renais ${ }^{1}$. Apesar do restabelecimento da função renal parcial ou total, os pacientes podem continuar apresentando complicações do quadro urêmico (alterações cardiovasculares e do sistema muscular) com repercussões sobre fibras musculares e do $\mathrm{SNA}^{2,3}$, culminando com possíveis limitações funcionais ${ }^{2}$.

As repercussões urêmicas estão associadas à diminuição da capacidade funcional, reduzindo em cerca de $50 \%$ a funcionalidade, ocasionando diminuição na tolerância ao exercício e prejuízo significativo na qualidade de vida e nas atividades cotidianas ${ }^{4,5}$.

Os mecanismos associados às alterações cardiovasculares sobre a atividade autonômica urêmica não foram completamente elucidados. No entanto, podem ser explicados pelo desequilíbrio autonômico relacionado à uremia, com aumento da atividade do sistema nervoso simpático (SNS) e redução da atividade parassimpática, representando forte indicador de risco de mortalidade cardíaca ${ }^{3,4,6,7}$.

A análise da variabilidade da frequência cardíaca (VFC) nos domínios do tempo e da frequência é reconhecidamente marcador diagnóstico, prognóstico e preditor de complicações, inclusive de mortalidade ${ }^{8}$, sendo de interesse para o estudo nessa população.

Considerando os efeitos da permanência dos sintomas urêmicos mesmo após o transplante renal, e esses resultando em diminuição da capacidade funcional e elevada morbimortalidade por problemas cardiovasculares dos pacientes, o presente estudo teve como objetivo avaliar a VFC e a capacidade funcional nos transplantados renais de acordo com o tempo de realização do transplante.

\section{Materiais e métodos}

Série de casos constituída por indivíduos transplantados renais acompanhados no ambulatório de um hospital terciário, no período de março a setembro de 2017, da Universidade Federal de Pernambuco.

O presente estudo foi aprovado pelo comitê de ética em pesquisa institucional (No. 1.884.578), de acordo com a Resolução 466/2012 do CONEP.

A amostra foi obtida por conveniência e composta por 14 indivíduos selecionados durante o período de realização do estudo.

Foram incluídos indivíduos transplantados renais com idade entre 18 e 59 anos, de ambos os sexos, com tempo de transplante igual ou superior a um ano, que não utilizassem medicamentos com capacidade de modificar o sistema nervoso autônomo.

Foram excluídos do estudo os indivíduos com hipertensão arterial não controlada e parâmetros hemodinâmicos instáveis, doenças osteomioarticulares, insuficiência cardíaca congestiva, infarto do miocárdio recente e angina instável.

Inicialmente, foram coletados os dados antropométricos (sexo, idade, índice de massa corpórea (IMC)) e a história clínica no momento da avaliação (comorbidades, tipo e tempo de transplante, tipo de TRS prévia ao transplante, uso de medicamentos e níveis séricos de creatinina, hemoglobina e hematócrito). Posteriormente, foi avaliado o nível de atividade física por meio do Questionário Internacional de Atividade Física versão curta (IPAQ) e instalado o Holter por 24 horas para o estudo da VFC. O teste de caminhada de 6 minutos (TC6min) foi realizado após a retirada do Holter.

\section{Nível de atividade física}

Avaliada por meio do International Physical Activity Questionnaire (IPAQ) versão curta9. Para este estudo, os participantes foram classificados em sedentários (realizavam nenhuma atividade 
ou que relatassem alguma atividade) ou ativos ('muito ativos', 'ativos' e irregularmente ativos).

\section{Variabilidade da frequência cardíaca}

Foi avaliada por meio do equipamento Holter Cardios ${ }^{\circledR}$, modelo Cardio Light (Cardio Sistemas Coml. Indl. Ltda, São Paulo, Brasil) por 24 horas. Para realização deste exame, foram afixados quatro eletrodos (manúbrio esternal, apêndice xifoide e entre a quinta e sexta costelas de ambos os lados).

Todas as informações digitais de monitorização foram armazenadas em um cartão de memória removível e analisadas posteriormente pelo software do aparelho. Os testes e análises dos resultados do exame foram interpretados, avaliados e laudados por um cardiologista devidamente habilitado para esse tipo de exame. Os índices da VFC foram analisados por meio de métodos lineares compostos pela análise dos domínios de tempo e de frequência.

O domínio de tempo expressa os resultados em unidade de tempo (milissegundos - ms) e mede cada intervalo R-R normal (batimentos sinusais) durante determinado intervalo de tempo, resultando nos seguintes parâmetros: SDNN - desvio padrão de todos os intervalos R-R no intervalo de tempo, este representa as atividades simpática e parassimpática; rMSSD - raiz quadrada da média do quadrado das diferenças entre intervalos R-R normais adjacentes, que representa a atividade parassimpática; $\mathrm{PNN}>50(\%)$ - porcentagem dos intervalos $\mathrm{R}-\mathrm{R}$ adjacentes com diferença de duração maior que $50 \mathrm{~ms}$ e representa a atividade parassimpática9 .

A análise no domínio frequência, obtendo a análise espectral da VFC, são medidas derivadas da análise da densidade do espectro de potência, que descreve a distribuição da densidade (variância) em função da frequência, a saber: Componente de Alta Frequência (HF) - com variação de 0,15 a $0,40 \mathrm{~Hz}$, que corresponde à modulação respiratória; Componente de baixa frequên- cia (LF) - com variação entre 0,04 e 0,15Hz, que é decorrente da ação conjunta dos componentes de modulação simpático e parassimpático (vagal) sobre o coração, com predominância do simpático, representando a atividade barorreflexa; Relação LF/HF - reflete as alterações absolutas e relativas entre os componentes simpático e parassimpático do SNA, caracterizando o balanço/equilíbrio simpático vagal sobre o coração; FC - constituída pela média dos valores instantâneos de FC em batimentos por minuto (bpm) $)^{4,6,7,10}$. Os indivíduos foram classificados como 'balanço autonômico normal' e 'depressão do tônus vagal' de acordo com os resultados da VFC.

\section{Teste de caminhada de 6 minutos (TC6min)}

O TC6min foi realizado de acordo com as normas da $\mathrm{ATS}^{11}$. Ao final do teste, foi registrada a distância percorrida (DP) em metros. Os valores preditos da DP foram calculados a partir do estudo de Enright e Sherrill ${ }^{12}$.

\section{Anólise estatística}

Os transplantados foram divididos em: grupo $<158$ meses e grupo $\geq 158$ meses, de acordo com a mediana do tempo de transplante renal obtido da amostra. A caracterização dos grupos foi apresentada por meio de média e intervalo de confiança e distribuição de frequência. $O$ comportamento de cada variável do estudo foi descrita individualmente conforme o grupo ao qual pertenciam, a fim de se verificar o comportamento dos indivíduos de acordo com o tempo de realização do transplante renal.

\section{Resultados}

Foram avaliados 14 transplantados renais. As características clínicas, antropométricas e laboratoriais dos indivíduos nos grupos estão descritas na tabela 1. 
Tabela 1: Características antropométricas, clínicas, laboratoriais, nível de atividade física, TC6m e de todos os transplantados renais do Estudo e divididos no grupo $<158$ meses e $\geq 158$ meses $(n=14)$.

\begin{tabular}{|c|c|c|}
\hline \multirow{2}{*}{ Variáveis } & $<158$ meses $(n=7)$ & $\geq 158$ meses $(n=7)$ \\
\hline & n (\%) / Mediana (IC 95\%) & n (\%) / Mediana (IC 95\%) \\
\hline Idade (anos) & $48(32,1-53)$ & $45(40,67-54,47)$ \\
\hline IMC $\left(\mathrm{Kg} / \mathrm{m}^{2}\right)$ & $26,8(22,5-30,4)$ & $22,5(19,9-26,6)$ \\
\hline \multicolumn{3}{|l|}{ Sexo } \\
\hline Masculino & $4(57,1 \%)$ & $5(71,4 \%)$ \\
\hline \multicolumn{3}{|l|}{ Transplante } \\
\hline Tempo de TX (meses) & $100(55,9-134,6)$ & $192(168,1-234,9)$ \\
\hline $\begin{array}{l}\text { Tempo de tratamento } \\
\text { anterior }\end{array}$ & $71(29,5-119,8)$ & $108(65,41-124,59)$ \\
\hline \multicolumn{3}{|l|}{ Exames Laboratoriais } \\
\hline Creatinina $(\mathrm{mg} / \mathrm{dL})$ & $1,6(1,2-2,5)$ & $1,5(1,1-2)$ \\
\hline Hemoglobina (g/dL) & $12,3(11,5-14,1)$ & $12,5(11,613,9)$ \\
\hline Hematócrito (\%) & $37(34,7-42,3)$ & $40(35,5-43,1)$ \\
\hline TFG $\left(\mathrm{ml} / \mathrm{min} / 1,73 \mathrm{~m}^{2}\right)$ & $52,80(27,07-64,64)$ & $49,50(38,84-80,20)$ \\
\hline \multicolumn{3}{|c|}{ Tipo de tratamento anterior } \\
\hline Hemodiálise (HD) & $7(100 \%)$ & $4(57,1 \%)$ \\
\hline$H D+D P$ & - & $3(42,9 \%)$ \\
\hline \multicolumn{3}{|l|}{ Comorbidades } \\
\hline Sim & 7 & 6 \\
\hline HAS & $7(100 \%)$ & $5(71,4 \%)$ \\
\hline HAS + DM & - & $1(14,2 \%)$ \\
\hline
\end{tabular}

Legenda: IMC = Índice de massa corporal; HD = Hemodiálise; DP = Diálise peritoneal; HAS = Hipertensão arterial sistêmica; DM = Diabetes Mellitus; IPAQ = Questionário Internacional de Atividade Física; TC6min= Teste de caminhada de 6 minutos; $\mathrm{TX}=$ Transplante Renal.

Fonte: Os autores.

te P5); e o tempo de realização do transplante renal variou entre 13 (paciente P3) e 154 meses (paciente P7). Quanto ao nível de atividade física, $100 \%$ das mulheres e $57,1 \%$ dos homens foram considerados ativos.

Todos os transplantados fizeram uso de imunossupressores e de outras medicações (Tabela 2).

Em relação ao desempenho no TC6min, pôde-se verificar que todos os transplantados apresentaram desempenho funcional superior a $60 \%$ do previsto, tendo o transplantado P7 obtido a melhor distância percorrida (88.02\% do previsto).

Quanto a VFC, $42,85 \%$ dos transplantados (pacientes P1, P5 e P7) apresentaram balanço autonômico e as menores frequências cardíacas. O comportamento das variáveis nos domínios do tempo (SDNN, rMSSD e PNN $>50 \%$ ) e da frequência (HF, LF, LF/ $\mathrm{HF})$ variaram consideravel-

As características individuais de cada transplantado renal encontram-se descritas nas tabelas 2 e 3, respectivamente, de acordo com os grupos: <158 meses (pacientes P1 a P7) e $\geq 158$ meses (pacientes P8 a P14).

\section{Grupo < 158 meses de transplante renal}

No grupo com tempo de transplante renal menor que 158 meses, pouco mais da metade foi constituída por homens (57,1\%). A TFG variou entre 20,50 (paciente P7) e $71,10 \mathrm{~mL} / \mathrm{min} / 1,73 \mathrm{~m}^{2}$ (paciente P4); o tempo de TRS prévio ao transplante, entre 5 (paciente P3) e 156 meses (pacien- mente entre eles (Tabela 2).

\section{Grupo $\geq 158$ meses de transplante renal}

O grupo acima de 158 meses de transplante renal foi constituído predominantemente por homens (71,4\%). Esse grupo apresentou menores valores de IMC (pacientes P8, P9, P11 e P14) em relação ao grupo com menor tempo. A TFG variou entre 29,20 (paciente P14) e $64,50 \mathrm{ml} /$ min $/ 1,73 \mathrm{~m}^{2}$ (paciente P12); o tempo de TRS prévio ao transplante, entre 18 (paciente P8) e 127 meses (paciente P13); e o tempo de realização do transplante renal variou entre 162 (paciente P9) e 
Tabela 2: Características antropométricas, clínicas, laboratoriais, parâmetros da VFC, nível de atividade física, TC6m dos transplantados renais com tempo de TX inferior a 158 meses

\begin{tabular}{|c|c|c|c|c|c|c|c|}
\hline Paciente & $\mathrm{P} 1$ & P2 & P3 & P4 & P5 & P6 & P7 \\
\hline Idade (anos) & 52 & 34 & 21 & 48 & 49 & 43 & 51 \\
\hline Sexo, M/F & $\mathrm{F}$ & $\mathrm{M}$ & $\mathrm{M}$ & $\mathrm{M}$ & $\mathrm{F}$ & $\mathrm{F}$ & $\mathrm{M}$ \\
\hline $\mathrm{IMC}, \mathrm{Kg} / \mathrm{m}^{2}$ & 33,5 & 23,9 & 26,8 & 24,8 & 29,4 & 20,0 & 27,0 \\
\hline \multicolumn{8}{|l|}{ Transplante } \\
\hline Tempo de TX (meses) & 113 & 95 & 13 & 100 & 108 & 84 & 154 \\
\hline \multicolumn{8}{|l|}{ Tipo de TX } \\
\hline Doador vivo & Não & Não & Sim & Sim & Não & Não & Não \\
\hline Doador cadáver & Sim & Sim & Não & Não & Sim & Sim & Sim \\
\hline Tempo de TRS (meses) & 84 & 36 & 5 & 71 & 156 & 108 & 63 \\
\hline Tipo de TRS & HD & $\mathrm{HD}$ & HD & HD & HD & HD & HD \\
\hline \multicolumn{8}{|l|}{ Comorbidades } \\
\hline HAS & Sim & Sim & Sim & Sim & Sim & Sim & Sim \\
\hline DM & Não & Não & Não & Não & Não & Não & Não \\
\hline \multicolumn{8}{|l|}{ Laboratoriais } \\
\hline Creatinina $(\mathrm{mg} / \mathrm{dL})$ & 1,5 & 1,6 & 1,5 & 1,2 & 1,9 & 2,3 & 3,3 \\
\hline TFG $\left(\mathrm{mL} / \mathrm{min} / 1,73 \mathrm{~m}^{2}\right)$ & 52,80 & 55,40 & 65,60 & 71,10 & 30,40 & 25,20 & 20,50 \\
\hline \multicolumn{8}{|l|}{ Medicações } \\
\hline Imumossupressores & Sim & Sim & Sim & Sim & Sim & Sim & Sim \\
\hline Anti-hipertensivos & Sim & Sim & Sim & Sim & Sim & Sim & Sim \\
\hline Outros & Não & Não & Sim & Sim & Não & Não & Não \\
\hline \multicolumn{8}{|l|}{ Imunossupressores } \\
\hline Azatioprina & Não & Não & Não & Não & Sim & Sim & Sim \\
\hline Ciclosporina & Não & Não & Não & Sim & Sim & Sim & Não \\
\hline Micofenolato de mofetil & Sim & Sim & Não & Não & Não & Não & Não \\
\hline Micofenolato de sódio & Não & Não & Sim & Não & Não & Não & Não \\
\hline Prednisona & Sim & Sim & Sim & Sim & Sim & Sim & Sim \\
\hline Tacrolimus & Sim & Sim & Sim & Não & Não & Não & Não \\
\hline \multicolumn{8}{|l|}{ IPAQ } \\
\hline Ativo & Sim & Não & Sim & Sim & Sim & Sim & Não \\
\hline Sedentário & Não & Sim & Não & Não & Não & Não & $\operatorname{Sim}$ \\
\hline DTC6min (metros) & 441 & 470 & 459 & 487 & 443 & 423 & 504 \\
\hline TC6min \%pred & 86,78 & 65,60 & 65,53 & 88,60 & 73,35 & 66,20 & 88,02 \\
\hline \multicolumn{8}{|l|}{ VFC } \\
\hline \multicolumn{8}{|l|}{ Domínio do tempo } \\
\hline SDNN & 121 & 113 & 84 & 127 & 162 & 110 & 105 \\
\hline rMSSD & 38 & 21 & 15 & 18 & 21 & 19 & 26 \\
\hline $\mathrm{PNN}>50 \%$ & 8,57 & 2,21 & 0,42 & 1,55 & 1,39 & 1,36 & 4,32 \\
\hline \multicolumn{8}{|l|}{ Domínio da Frequência } \\
\hline $\mathrm{HF}$ & 160,1 & 244,1 & 35,9 & 107,6 & 68,5 & 94,8 & 135,04 \\
\hline $\mathrm{LF}$ & 112,3 & 308,6 & 220,2 & 307,0 & 203,3 & 122,0 & 442,8 \\
\hline Relação LF/HF & 0,98 & 2,68 & 7,81 & 5,0 & 5,22 & 2,45 & 3,96 \\
\hline FC (bpm) & 53,3 & 84,6 & 99,8 & 86,6 & 60,8 & 78,7 & 64,1 \\
\hline \multicolumn{8}{|l|}{ Modulação autonômica } \\
\hline Balanço & Sim & Não & Não & Não & Sim & Não & Sim \\
\hline Desbalanço & Não & Sim & Sim & Sim & Não & Sim & Não \\
\hline
\end{tabular}

Legenda: $\mathrm{M}$ = masculino; F = feminino; IMC = Índice de massa corporal; Tx = Transplante renal; TRS = Terapia renal substitutiva; HD = Hemodiálise; DP = Diálise peritoneal; HAS = Hipertensão arterial sistêmica; DM = Diabetes Mellitus; TFG= Taxa de filtração glomerular; IPAQ = Questionário Internacional de Atividade Física; DTC6 = Distância percorrida no Teste de Caminhada de 6 minutos; VFC = Variabilidade da frequência cardíaca; Balanço = Balanço autonômico normal; Desbalanço = depressão do tônus vagal; SDNN: desvio padrão de todos os intervalos R-R no intervalo de tempo; rMSSD: raiz quadrada da média do quadrado das diferenças entre intervalos R-R normais adjacentes; PNN>50\%: porcentagem dos intervalos RR adjacentes com diferença de duração maior que 50ms; HF: alta frequência; LF: baixa frequência ; relação HF/LF: alta frequência/baixa frequência; FC: frequência cardíaca.

Fonte: Os autores. 
269 meses (paciente P12). Quanto ao nível de atividade física, $100 \%$ das mulheres e $20 \%$ dos homens (paciente P11) foram considerados ativos.

Todos os transplantados fizeram uso de imunossupressores e de outras medicações (Tabela 3).

Em relação ao TC6min, pode-se observar que apenas um transplantado apresentou desempenho funcional inferior a $50 \%$ do previsto (paciente P14). Esse transplantado também apresentou o menor IMC do grupo $\left(19,2 \mathrm{Kg} / \mathrm{m}^{2}\right)$, a menor TFG $\left(29,20 \mathrm{ml} / \mathrm{min} / 1,73 \mathrm{~m}^{2}\right)$ e era sendentário.

Quanto a VFC, 71,43\% dos transplantados (pacientes P8, P9, P11, P12 e P13) apresentaram balanço autonômico. O comportamento das variáveis nos domínios do tempo (SDNN, rMSSD e $\mathrm{PNN}>50 \%$ ) e da frequência (HF, LF, LF/HF) variou consideravelmente entre eles, conforme pode ser observado na tabela 3).

\section{Discussão}

Até o presente momento, não foram encontrados estudos que tivessem avaliado o comportamento da VFC e da capacidade funcional em transplantados renais ao longo do tempo de transplante (TX). O presente estudo observou que a maior parte dos indivíduos do grupo com tempo de TX superior a 158 meses apresentou balanço autonômico e, em relação ao desempenho funcional no TC6min, a maior parte dos transplantados do estudo superou $60 \%$ do previsto para a distância percorrida.

Em nosso estudo, a TFG em torno e abaixo de $60 \mathrm{~mL} / \mathrm{min} / 1,73 \mathrm{~m}^{2}$ foi observada na maior parte dos transplantados, refletindo, possivelmente, funcionamento precário do enxerto renal e manutenção do quadro urêmico crônico. As anormalidades decorrentes da uremia crônica na DRC podem permanecer mesmo após a realização do TX renal, embora os efeitos sobre a VFC possam melhorar entre 3 e 6 meses após a realização do transplante ${ }^{13}$. No entanto, considerando o tempo de TX dos indivíduos de nosso estudo e a apresentação de relativa variância nos domínios do tempo e da frequência entre eles, é possível que a presença de toxinas urêmicas possa contribuir para o aumento da atividade simpática ${ }^{14,15}$.

Sobre esse ponto, observamos ainda que os transplantados com menor tempo de TX apresentaram valores referentes ao SDNN mais baixos em relação ao grupo com maior tempo, sugerindo, possivelmente, presença de menor atividade parassimpática e evidenciando disfunção autonômica cardíaca ${ }^{10}$. Para o rMSSD e o PNN $>50 \%$, foi observada variância considerável entre os transplantados, especialmente para o grupo com maior tempo de TX. Rubinger et al (1999) acompanharam o comportamento da VFC de 4 transplantados renais com até 10 anos de TX e verificaram manutenção, melhora e piora da VFC e consideraram a possibilidade do tempo de HD anterior ao TX contribuir para esse comportamento ${ }^{15}$. Os transplantados de nosso estudo apresentaram entre 100 e 192 meses de HD antes do TX, média superior ao encontrado no estudo acima citado (42 meses), e também apresentaram comportamento variado entre os domínios do tempo e da frequência.

Acreditamos que a alteração da VFC nos pacientes transplantados seja multifatorial ${ }^{15} \mathrm{e}$ que o uso de imunossupressore ${ }^{14}$, a presença de hipertensão arterial ${ }^{16}$ e o tempo de HD prévio ao transplante ${ }^{14}$ dos participantes de nosso estudo possam estar concorrendo para a baixa VFC encontrada.

Em nosso estudo, cerca de $43 \%$ dos transplantados renais eram sedentários. Embora a diminuição do nível de atividade física nesse grupo de pacientes seja frequente, a persistência da uremia ${ }^{14}$, o uso de corticosteroides ${ }^{17}$ e de imunossupressores ${ }^{18}$, associados à presença de comorbidades como hipertensão, obesidade e diabetes ${ }^{19}$, têm sido descritos como prováveis fatores que podem contribuir para o sedentarismo e o baixo desempenho funcional de transplantados renais. Para os transplantados com maior tempo de TX se desconhece sobre esse comportamento ${ }^{19}$. Os participantes de nosso estudo apresentaram tempo de TX superior a 100 meses 
Tabela 3: Características antropométricas, clínicas, laboratoriais, parâmetros da VFC, nível de atividade física, TC6m dos transplantados renais com tempo de TX superior ou igual a 158 meses

\begin{tabular}{|c|c|c|c|c|c|c|c|}
\hline Paciente & P8 & P9 & P10 & P11 & $\mathrm{P} 12$ & P13 & P14 \\
\hline Idade (anos) & 59 & 43 & 44 & 56 & 48 & 45 & 38 \\
\hline Sexo, M/F & $\mathrm{F}$ & $\mathrm{M}$ & $\mathrm{M}$ & $\mathrm{M}$ & $\mathrm{M}$ & $\mathrm{F}$ & $\mathrm{M}$ \\
\hline $\mathrm{IMC}, \mathrm{Kg} / \mathrm{m}^{2}$ & 20,8 & 20,3 & 24,5 & 22,5 & 28,1 & 27,7 & 19,2 \\
\hline \multicolumn{8}{|l|}{ Transplante } \\
\hline Tempo de TX (meses) & 200 & 162 & 180 & 180 & 269 & 229 & 192 \\
\hline \multicolumn{8}{|l|}{ Tipo de TX } \\
\hline Doador vivo & Não & Sim & Não & Não & Sim & Não & Não \\
\hline Doador cadáver & Sim & Não & Sim & Sim & Não & Sim & Sim \\
\hline Tempo de TRS (meses) & 18 & 36 & 108 & 84 & 76 & 127 & 114 \\
\hline Tipo de TRS & $H D+D P$ & HD & HD & HD & $H D+D P$ & HD & $H D+D P$ \\
\hline \multicolumn{8}{|l|}{ Comorbidades } \\
\hline HAS & Sim & Sim & Sim & Sim & Sim & Sim & Não \\
\hline DM & Não & Não & Não & Não & Não & Não & Não \\
\hline \multicolumn{8}{|l|}{ Laboratoriais } \\
\hline Creatinina (mg/dL) & 1,0 & 1,8 & 1,8 & 1,5 & 1,3 & 1,3 & 2,6 \\
\hline TFG $\left(\mathrm{mL} / \mathrm{min} / 1,73 \mathrm{~m}^{2}\right)$ & 61,60 & 45,10 & 44,80 & 51,30 & 64,50 & 49,50 & 29,20 \\
\hline \multicolumn{8}{|l|}{ Medicações } \\
\hline Imumossupressores & Sim & Sim & Sim & Sim & Sim & Sim & Sim \\
\hline Anti-hipertensivos & Sim & Sim & Sim & Sim & Sim & Sim & Não \\
\hline Outros & Sim & Não & Não & Não & Sim & Não & Não \\
\hline \multicolumn{8}{|l|}{ Imunossupressores } \\
\hline Azatioprina & Sim & Sim & Não & Sim & Sim & Sim & Sim \\
\hline Ciclosporina & Sim & Sim & Não & Não & Sim & Sim & Não \\
\hline Micofenolato de mofetil & Não & Não & Sim & Não & Não & Não & Não \\
\hline Micofenolato de sódio & Não & Não & Não & Não & Não & Não & Não \\
\hline Prednisona & Sim & Sim & Sim & Sim & Sim & Sim & Sim \\
\hline Tacrolimus & Não & Não & Não & Sim & Não & Não & Sim \\
\hline \multicolumn{8}{|l|}{ IPAQ } \\
\hline Ativo & Sim & Não & Não & Sim & Não & Sim & Não \\
\hline Sedentário & Não & Sim & Sim & Não & Sim & Não & Sim \\
\hline DTC6min (metros) & 438 & 458 & 558 & 572 & 510 & 383 & 235 \\
\hline TC6min \%pred & 82,86 & 68,24 & 101,04 & 94,17 & 91,44 & 66,10 & 41,87 \\
\hline \multicolumn{8}{|l|}{ VFC } \\
\hline \multicolumn{8}{|l|}{ Domínio do tempo } \\
\hline SDNN & 182 & 146 & 103 & 177 & 107 & 159 & 101 \\
\hline rMSSD & 74 & 44 & 10 & 138 & 21 & 29 & 18 \\
\hline PNN $>50 \%$ & 19,7 & 16,99 & 0,14 & 15,34 & 2,11 & 4,49 & 1,25 \\
\hline \multicolumn{8}{|l|}{ Domínio da Frequência } \\
\hline $\mathrm{HF}$ & 1027,6 & 480,3 & 17,6 & 3491,2 & 130,8 & 187,8 & 128,1 \\
\hline $\mathrm{LF}$ & 2564,7 & 1886,0 & 239,2 & 1468,3 & 365,8 & 1080,1 & 381,5 \\
\hline Relação LF/HF & 1,96 & 4,20 & 12,64 & 4,21 & 4,34 & 8,69 & 5,52 \\
\hline FC (bpm) & 67,84 & 66,04 & 84,32 & 72,28 & 70,88 & 91,50 & 81,25 \\
\hline \multicolumn{8}{|l|}{ Modulação autonômica } \\
\hline Balanço & Sim & Sim & Não & Sim & Sim & Sim & Não \\
\hline Desbalanço & Não & Não & Sim & Não & Não & Não & Sim \\
\hline
\end{tabular}

Legenda: $\mathrm{M}$ = masculino; $\mathrm{F}$ = feminino; IMC = Índice de massa corporal; $\mathrm{Tx}=$ Transplante renal; TRS = Terapia renal substitutiva; $\mathrm{HD}=$ Hemodiálise; DP = Diálise peritoneal; HAS = Hipertensão arterial sistêmica; DM = Diabetes Mellitus; TFG= Taxa de filtração glomerular; IPAQ = Questionário Internacional de Atividade Física; DTC6min = Distância percorrida no Teste de Caminhada de 6 minutos; VFC = Variabilidade da frequência cardíaca; Balanço = Balanço autonômico normal; Desbalanço = depressão do tônus vagal; SDNN: desvio padrão de todos os intervalos R-R no intervalo de tempo; rMSSD: raiz quadrada da média do quadrado das diferenças entre intervalos R-R normais adjacentes; PNN $>50 \%$ : porcentagem dos intervalos RR adjacentes com diferença de duração maior que $50 \mathrm{~ms}$; HF: alta frequência; LF: baixa frequência ; relação HF/LF: alta frequência/baixa frequência; FC: frequência cardíaca.

Fonte: Os autores 
e esse achado foi acompanhado de níveis séricos de creatinina relativamente elevados e TFG inferior a $60 \mathrm{~mL} / \mathrm{min} / 1,73 \mathrm{~m}^{2}$, sugestivos de declínio da função renal e do estado urêmico característico da DRC.

De acordo com o desempenho funcional dos transplantados de nosso estudo, pode-se verificar que a maior parte se manteve acima de $60 \%$ predito para a distância percorrida no TC6min, a despeito do nível de atividade física, do uso de corticosteroide e de imunossupressores. O uso contínuo de agentes imunossupressores e de corticosteroides é capaz de induzir miopatia, fraqueza muscular, inibição de síntese e aumento do catabolismo proteico, provocando reduzida capacidade funcional ${ }^{17,18}$. No entanto, explicar o comportamento individual do transplantado se torna uma tarefa difícil.

Entre os indivíduos do grupo com menor tempo de TX, observamos que o P7 apresentou o melhor desempenho funcional $(88,02 \%$ da distância prevista), ao mesmo tempo em que apresentou sobrepeso, nível de creatinina sérica elevada e baixa TFG, maior tempo de TX do grupo, inatividade e balanço autonômico.

O transplantado P14, do grupo com maior tempo de TX, apresentou o pior desempenho funcional do grupo (41,87\% da distância prevista). Esse paciente apresentou o menor IMC (19,2 $\left.\mathrm{Kg} / \mathrm{m}^{2}\right)$, nível de creatinina sérica elevada e baixa TFG, desbalanço autonômico e inatividade.

Esse estudo reflete diferenças individuais entre os transplantados renais e, a despeito do tempo de transplante, podemos observar que os diferentes perfis antropométricos, laboratoriais e de histórico pregresso da DRC podem estar contribuindo para que se comportem diferentemente em relação à VFC e ao desempenho funcional no TC6min. Reconhecemos que o reduzido número de indivíduos avaliados, limitados a um único centro transplantador, pode ter dificultado não apenas o uso da inferência estatística, bem como de apresentarmos uma conclusão mais precisa acerca do tema.

Ao mesmo tempo, esse estudo chama a atenção para a importância da apresentação do individual, que expressa a real condição desse transplantado, permitindo que a avaliação realizada possa considerar detalhes que talvez não pudessem ser visualizados com o uso da média e do desvio padrão.

Com essa perspectiva em mente, esta série de casos chama a atenção para a presença de diferenças de comportamento clínico e funcional entre os transplantados renais de acordo com o tempo de realização do TX. Diante da possibilidade de diferenças individuais, o profissional de saúde pode estabelecer estratégias de intervenção, por meio do exercício físico, direcionadas para cada caso específico, objetivando resultados efetivos para a redução de eventos cardiovasculares, muito frequentes nesse grupo de pacientes, e também a redução do sedentarismo entre esses indivíduos.

\section{Referências}

1. Schlaich MP. Sympathetic activation in chronic kidney disease: out of the shadow. Hypertension. 2011; 57(4): 683-685.

2. Deus LA, Simões HG, Neves RVP, Souza MK, Moraes MR, Navarro F, et al. Associação da variabilidade da frequência cardíaca e estress oxidativo: o papel do exercício físico. Rev Bras de Presc e Fis do Exerc. 2017; 11(66): 366-376

3. Rodrigues $\mathrm{PH}$, Oliveira MB, Cazelato L, Chagas EFB, Quitrio RJ. A influência dos fatores de risco para doenças cardiovasculares sobre a modulação autonômica cardíaca. Rev Aten Saúde. 2016; 14(48): 34-40.

4. Kouidi E, Vergoulas G, Anifanti M, Deligiannis A. A randomized controlled trial of exercise training on cardiovascular and autonomic function among renal transplant recipients. Nephrol Dial Transplant. 2013; 28(5): 1294-1305.

5. Cury JL, Brunetto AF, Aydos RD. Efeitos negativos da insuficiência renal crônica sobre a função pulmonar e a capacidade funcional. Rev Bras Fisioter. 2010; 14(2): 91-98.

6. Vanderlei LCM, Pastre CM, Hoshi RA, Carvalho TD, Godoy MF. Noções básicas de variabilidade da frequência cardíaca e sua aplicabilidade clínica. Rev Bras Cir Cardiovasc. 2009; 24(2): 205-217. 
7. Marães VRFS. Frequência cardíaca e sua variabilidade: análises e aplicações. Rev Andal Med Deporte. 2010; 3(1): 33-42.

8. La Rovere MT, Pinna GD, Maestri R, Barlera S, Bernardinangeli M, Veniani M, Nicolosi GL, et al. Autonomic markers and cardiovascular and arrhythmic events in heart failure patients: still a place in prognostication? Data from the GISSI-HF trial. Eur J Heart Fail. 2012; 14(12): 1410-1419.

9. Matsudo S, Araújo T, Matsudo V, Andrade D, Andrade E, Oliveira C, Braggion G. Questionário internacional de atividade física (IPAQ): estudo de validade e reprodutibilidade no Brasil. Rev Bras Ativ Fis Saúde. 2001; 6(2): 5-12.

10. Task Force of the European Society of Cardiology of the North American Society of Pacing Electrophysiology. Heart rate variability standards of measurement, physiological interpretation, and clinical use. Eur Heart J. 1996; 93(5): 354-381.

11. ATS Committee Standards for Clinical Pulmonary Function Laboratories. ATS Statement. Guidelines for the six-minute walk test. Am J Respir Crit Care Med. 2002; 166(1): 111-117.

12. Enright PL, Sherrill DL. Reference equations for the six-minute walk in healthy adults. Am J Respir Crit Care Med. 1998; 158(5):1384-1387
13. Yıldız A, Sever MS, Demirelb S, Akkaya V, Türka $\mathrm{S}$, Türkmena A, et al. Improvement of uremic autonomic dysfunction after renal transplantation: a heart rate variability study. Nephron. 1998; 80:57-60.

14. Hausberg M, Kosch M, Harmelink P, Barenbrock, M, Helge $\mathrm{H}$, Kisters $\mathrm{K}$ et al. Sympathetic nerve activity in end-stage renal disease. Circulation. 2002; 106: 1974-1979

15. Rubinger D, Sapoznikov D, Pollak A, Popovtzer MM, Luria $\mathrm{MH}$. Heart rate variability during chronic hemodialysis and after renal transplantation: studies in patients without and with systemic amyloidosis. J Am Soc Nephrol. 1999. 10(9): 19721981.

16. Koomans HA, Ligtenberg G. Mechanisms and consequences of arterial hypertension after renal transplantation. Transplantation. 2001; 72(6): S9-S12.

17. Kouidi EJ. Central and peripheral adaptations to physical training in patients with end-stage renal disease. Sports Med 2001; 31 (9): 651-665.

18. McKenzie K-J L, McKenzie D C, Yoshida E M. Solid organ transplant recipients: clinical considerations in the application of exercise. Br J Sports Med 2015; 49:76-78.

19. Bellizzi V, Cupisti A, Capitanini A, Calella P, D'Alessandro C. Physical Activity and Renal Transplantation. Kidney Blood Press Res 2014; 39:212-219. 